ney and renal transplantation units have a serious effect on the morale of members of the unit, the laboratories and the hospital. The repercussions inevitably lead to problems in clinical management, recruitment of staff, administrative and financial difficulties. Indeed, hepatitis affects the whole future of renal dialysis and transplant programmes. The results of the deliberations of Lord Rosenheim's committee, appointed by the Department of Health and Social Security to consider these vexed problems, are therefore awaited with great interest.

\section{DEEP SEA FISH}

\section{Toothless Maturity}

from our Marine Vertebrate Correspondent

THE deep sea fishes of the genus Anotopterus are usually described as distinctive on account of the remarkably long palatine teeth in their elongate jaws. The capture in 1961 by RRS Discovery II in Madeiran waters of an apparent Anotopterus which was completely toothless, as recently reported by G. E. Maul (Bocagiana (Museu Municipal do Funchal), No. 28, 1; 1971), is therefore of some interest. The specimen described by Maul was taken in an Isaacs-Kidd Midwater trawl, SSE of Funchal, fished at $2,500 \mathrm{~m}$, although as some of the thirty-three species of fish caught at this station are near-surface living species and this net did not have a closing device, it is possible that the specimen of Anotopterus was caught in more shallow water than this during hauling.

Anotopterus pharao is the only known species of the genus, and it has been found widely distributed in both the Atlantic and Pacific Oceans. It is described as a long thin fish, with pointed beak-like jaws and a long head; its large teeth are distinctive, and in coloration it is dark on the back with metallic golden tints on the sides. Specimens up to $87 \mathrm{~cm}$ have been described. The Madeiran specimen, which measures 76 $\mathrm{cm}$, is referred to $A$. pharao by Maul in spite of its lack of teeth, and slight variation in colour, because he found on dissection that it is a mature fish with enormously developed gonads. As Maul points out, although a large number of Anotopterus have been caught and described, this is the first sexually mature specimen to have been noticed. He concludes that in this species sexual maturity is accompanied by a complete loss of teeth, and a consequent atrophy of the gut, for in his specimen the gut is thin walled, empty and has no internal parasites.

Atrophy of the gut and degeneration of jaws and head bones are known in other fishes. A notable example is the Atlantic salmon (Salmo salar) in which the head bones become decalcified as the gonads reach a state of maturity. The salmon, however, does not completely lose its dentition, but cases of reduction in the number of teeth or gill rakers are known in other deep sea iniomous fishes.

Maul suggests that the causative factor of toothlessness may be a cessation of tooth replacement (which is a continuous process in Anotopterus and other fish species). $\mathrm{He}$ also discusses the possibility that it might be accounted for by vitamin $\mathrm{D}$ deficiency in the deeper sea interfering with the availability of lime salts, a thesis put forward by Parr (Bull. Bingham Oceanogr. Coll., 3 (7), $1 ; 1937)$ to account for the frequency of poorly calcified skeletons in deep sea fishes. The view that poorly calcified fishes are necessarily deprived fishes seems unfortunate, for in the deep sea a light skeleton, and near neutral buoyancy, may be a positive advantage to the conservation of energy. Toothlessness in sexually mature Anotopterus pharao may be accounted for by a need to conserve basic materials for gonad development, as well as a compensating reduction of weight in skeletal material as the gonads grow.

A curious feature in the discovery of a sexually mature Anotopterus off Madeira is that according to the known distribution of this species in the northern hemisphere the larger known specimens have been captured in high latitudes, the smaller ones nearest the equator. The inference drawn by other workers was that the species migrates regularly northwards as it grows, and Maul now proposes that a reverse migration must be made by the maturing adults to bring them back into tropical (and Madeiran) waters to spawn. This is a possibility, and might in time be confirmed by the capture of large, maturing Anotopterus in the area intervening between the tropics and Arctic seas. On the other hand, the evidence to date can be equally well interpreted to show that a small proportion of the Anotopterus population moves northwards, outside the area of normal distribution for the species, grows to a larger size but never reaches sexual maturity. As other deep sea fishes behave in this way, it would not be surprising if this did not occur also in Anotopterus.

\title{
Part Sequencing of Viral Messengers
}

IN Nature New Biology next Wednesday (January 26) Nichols, Hay and Joklik report the sequence of the first twenty-five residues at the $5^{\prime}$ terminal end of a class of reovirus messenger RNAs-molecules which are translated in eukaryotic cells. Perhaps the most notable feature of this sequence is the absence of any chain initiation codon ; apparently at least four of the ten reovirus messenger RNAs, like the genome and messenger RNAs of the RNA bacteriophages, start with a run of bases which is not translated and which presumably functions either as a ribosome attachment site and/or provides recognition signals for RNA replicase.

Reovirus has a most curious genome ; not only is it made of double stranded RNA but it also comprises ten discrete segments which are classified by size into large, medium and small classes. Each segment is transcribed into a single messenger RNA and at least eight of these ten molecules are translated as monocistronic messengers. Reovirus cores, obtained by removing the outer capsid from intact virions, transcribe in vitro all ten segments of the genome at a rate of about 600 bases per minute at $37^{\circ} \mathrm{C}$. By cooling to $31^{\circ} \mathrm{C}$ and starving for ATP Nichols and his colleagues cut this rate of synthesis so that the four molecules of the small class are synthesized preferen- tially at a rate of about 40 bases per minute. Under these conditions, by feeding suitably labelled nucleoside triphosphates, they managed to obtain a mixture of the four sorts of small reovirus messengers labelled at their $5^{\prime}$ termini. The sequence of the first twenty-five bases was then analysed by conventional methods.

All four molecules apparently begin with the same sequence (p)ppGpCpCpApUpUpUpUpUpGpCpUp(C,U)UpCpCpApGpApCpGpUpUpGp. Nichols et al. make the following comments about this sequence. The lability of the 5' phosphate residue presumably reflects the presence of nucleoside triphosphatases in the viral cores; there is no initiation codon; the second base is a cytidylate residue according to Nichols et al. although Banerjee et al. (Nature New Biology, 230, $169 ; 1971$ ) have reported a penultimate uridylate residue ; the sequence of the first nine bases of these reovirus molecules is the same as that between positions 48 to 56 of R17 and f2 RNA bacteriophage RNA.

When more of these reovirus molecules have been sequenced the significance and extent of such similarities may emerge; it will be particularly interesting to know the sequence of the reovirus molecules adjacent to the initiation site. 